\title{
PKA/CREB regulates the constitutive promoter activity of the USP22 gene
}

\author{
JIANJUN XIONG ${ }^{1,2}$, XIAOOU ZHOU ${ }^{1}$, ZHEN GONG $^{1}$, TING WANG ${ }^{1}$, \\ $\mathrm{CHAO}_{\mathrm{ZHANG}}{ }^{3}$, XIAOYUAN XU ${ }^{1,2}$, JIANYUN LIU ${ }^{2}$ and WEIDONG $\mathrm{LI}^{2}$ \\ ${ }^{1}$ College of Basic Medical Science, and ${ }^{2}$ Key Laboratory of Jiangxi Province for the Systems Bio-Medicine, \\ Jiujiang University, Jiujiang, Jiangxi 332000; ${ }^{3}$ The Maternal and Child Health Hospital \\ of Jiujiang City, Jiujiang, Jiangxi 332000, P.R. China
}

Received September 3, 2014; Accepted November 14, 2014

DOI: $10.3892 /$ or.2015.3740

\begin{abstract}
The human ubiquitin-specific processing enzyme 22 (USP22) plays a crucial role in regulating cell cycle processes and its overexpression has been linked to tumor progression. However, the mechanisms leading to USP22 transcriptional activation in human cancer cells are still unclear. Previously, we characterized the 5'-flanking sequence of the human USP22 gene and found a potential CREB/ATF binding site within the basic promoter region. The present study found that this site was required for constitutive USP22 transcriptional activity in HeLa and HepG2 cells. Chromatin immunoprecipitation assay confirmed that CREB interacted with this site. siRNA knockdown of CREB decreased USP22 transcriptional activation and endogenous expression, whereas CREB overexpression did not affect transcriptional levels. Furthermore, USP22 promoter activity and expression were decreased by inhibiting PKA with $\mathrm{H}-89$, but were not responsive to forskolin induction. All of these results demonstrate that PKA/CREB is involved in the regulation of constitutive promoter activity of the USP22 gene.
\end{abstract}

\section{Introduction}

The human ubiquitin-specific processing enzyme 22 (USP22) gene, which is located on chromosome 17 and consists of 14 exons, belongs to the deubiquitinating enzyme superfamily. Its transcription product is a 525 -amino acid polypeptide that is ubiquitously expressed in human adult tissues, including the heart and skeletal muscle, and is regularly expressed during early embryogenesis in mice (1). Recently, elevated levels of USP22 have been found in most types of human cancers, including colorectal (2), gastric (3) and breast cancer (4), suggesting a crucial role in tumorigenesis.

Correspondence to: Professor Weidong Li, Key Laboratory of Jiangxi Province for the Systems Bio-Medicine, Jiujiang University, 17 Lufeng Road, Jiujiang, Jiangxi 332000, P.R. China

E-mail: lwd626518@163.com

Key words: PKA, CREB, USP22, promoter
Structurally, USP22 contains a C-terminal peptidase domain, which functions as an ubiquitin hydrolase and an $\mathrm{N}$-terminal zinc finger motif, mediating other protein associations. As the key subunit of the human SAGA (hSAGA) transcriptional coactivator, USP22 functions as a regulatory activator governing the expression of several genes related to cancer and proliferation (5). Furthermore, USP22 has been shown to be required for the transcription of target genes regulated by the Myc oncoprotein, including cyclin D2, CAD, MTA1, with its downregulation leading to cell-cycle arrest $(5,6)$. USP22 has also been shown to deubiquitinate intracellular proteins, including a shelterin protein (7), a histone deacetylase (Sirt1) (8), and the far upstream elementbinding protein 1 (9). Murine studies have shown that Usp22 also regulates embryonic stem cell differentiation (10). Due to its extensive role in cancer progression, USP22 is considered a putative cancer stem cell marker and may serve as a novel cancer therapeutic target. However, the mechanisms leading to USP22 transcriptional activation, particularly in human cancer cells, are still unknown.

Previously, the USP22 promoter was cloned and characterized in HeLa cells by generating a series of $5^{\prime}$ deletions, to reveal that the -210 to +52 region contains the basal promoter activity (11). The TFSEARCH program was used to analyze this region and revealed the presence of a putative CREB binding site. However, the expressional role of this site in relation to USP22 promoter activity, or the role of putative transcription factor(s) binding, has not been established. Thus, the aim of the present study was to investigate the role of the CREB binding site in relation to USP22 transcriptional activity and identify any associated transcription factors. This study also aimed to characterize any upstream pathways regulating USP22 transcription through this site. Overall, the present study provides evidence to delineate the molecular mechanism governing human USP22 gene expression.

\section{Materials and methods}

Cell culture. Human cervical carcinoma (HeLa) cells and hepatocellular carcinoma (HepG2) cells were obtained from the American Tissue Culture Collection (ATCC; Manassas, VA, USA) and cultured in Dulbecco's modified Eagle's medium 
(DMEM; Invitrogen, Carlsbad, CA, USA) supplemented with $10 \%$ fetal bovine serum (FBS) at $37^{\circ} \mathrm{C}$ in a $5 \% \mathrm{CO}_{2}$ and $95 \%$ air incubator.

Plasmid constructs and cloning. Site-directed mutagenesis to inactivate the CREB binding site was carried out within the p-210 construct containing 262 bp of the 5'-flanking sequence of the USP22 gene using the MutanBEST kit (Takara, Shiga, Japan) according to the manufacturer's protocols. The following primers: 5'-GTagCGTAATCTCCGTCCGC-3' (sense) and 5'-CCCCCCCCCCCCTCCCGGCC-3' (antisense) were utilized and the mutation was confirmed by DNA sequencing.

CREB-1 full-length cDNA was obtained by reverse transcription PCR (RT-PCR). Briefly, total RNA was obtained from HeLa cells and reverse-transcribed with SuperScript ${ }^{\mathrm{TM}}$ II primed by oligo(dT) ${ }_{15}$ (Takara). cDNA products were amplified using the following PCR primers: 5'-AGGATCCATGACCATGGAATCTGGAGCC-3' (sense) and 5'-GGAATTCTTAATCTGATTTGTGGCAGTA-3' (antisense), digested with EcoRI and BamHI, and inserted into pCDNA 3.1(+).

Transfection and the dual luciferase reporter assay. Cells were plated in 24-well plates $24 \mathrm{~h}$ before transfection, with each well treated with $0.5 \mu \mathrm{g}$ of promoter construct, $0.1 \mu \mathrm{g}$ of pRL-TK (Promega, Madison, W, USA), and Lipofectamine 2000 (Invitrogen). All transfection experiments were repeated five times, with cells lysed $24 \mathrm{~h}$ after transfection and lysates used for the dual luciferase assays (Promega) according to the manufacturer's protocol. The normalized luciferase activity was expressed as a ratio of firefly luciferase activity to Renilla luciferase for each sample.

Chromatin immunoprecipitation (ChIP) assay. ChIP assays were performed using the EZ-ChIP kit according to the manufacturer's instructions (Pierce, Rockford, IL, USA). Approximately $10^{8}$ cells were fixed by adding formaldehyde to a final concentration of $1 \%$ and incubated for $30 \mathrm{~min}$ at room temperature with modest shaking. Thereafter, the cells were washed twice with cold phosphate-buffered saline, the pellet was resuspended and lysed, and the nuclei were isolated and sonicated until the chromatin had an average length of 400-500 bp. Following centrifugation, the supernatant was incubated with $1 \mu \mathrm{g}$ of anti-CREB1 antibody or rabbit IgG overnight at $4^{\circ} \mathrm{C}$ for immunoprecipitation. The following day, magnetic protein-G beads were added and further incubated at $4^{\circ} \mathrm{C}$ for $1 \mathrm{~h}$. After appropriate washing, the antibody-transcription factor-DNA complex was eluted from the beads, formaldehyde cross-links were reversed, and proteins were digested with proteinase $\mathrm{K}$ at $67^{\circ} \mathrm{C}$ overnight. DNA was purified and used for PCR with the primers, 5'-GGGCGGGCCCTCGTTAGCTT-3' and 5'-GGAGGCTG CAAGGCAGGCAC-3'.

RNA interference. One day before transfection, HeLa cells were plated in 24 -well plates at a density of $5 \times 10^{4}$ cells/well, and then transfected with $20 \mathrm{nM}$ of siControl Non-Targeting siRNA duplex (siControl) or human CREB1-specific siRNA duplex (sc-29281; Santa Cruz Biotechnology, Santa Cruz,
CA, USA), using an siRNA reagent system (Santa Cruz Biotechnology) according to the manufacturer's instructions. After $24 \mathrm{~h}$, the siRNA-transfected cells were harvested for real-time PCR or transfected with p-210 for the luciferase assay.

Quantitative real-time PCR (qRT-PCR). Cells were grown to confluency and RNA was extracted using TRIzol ${ }^{\circledR}$ reagent (Invitrogen). cDNA was synthesized from $1 \mu \mathrm{g}$ of total RNA using SuperScript II reverse transcriptase (Invitrogen) and random hexamers in a total volume of $20 \mu 1$ according to the manufacturer's instructions. The GAPDH gene was used as an endogenous control to normalize for RNA loading. qRT-PCR was performed using the SYBR-Green PCR Master Mix (Toyobo, Osaka, Japan) on an ABI 7500 Real-Time PCR System (Applied Biosystems, Foster City, CA, USA), with USP22 primers as previously described and validated (11).

Western blot analysis. Cells were lysed with 1X SDS sample buffer, and the protein was separated by $10 \%$ SDS-PAGE, and electroblotted onto a nitrocellulose membrane. The membrane was then incubated with the anti-USP22 antibody (Santa Cruz Biotechnology), the anti-CREB1 antibody (Santa Cruz Biotechnology), or the anti-GAPDH antibody (Santa Cruz Biotechnology) and visualized using the Pierce ECL system according to the manufacturer's instructions.

Statistical analysis. Data are presented as mean \pm SEM. Statistical differences between sample means were determined using an unpaired, two-tailed Student's t-test, with $\mathrm{P}<0.05$ deemed significant.

\section{Results}

CREB binding site mutation decreases USP22 promoter activity. Analysis of the basic USP22 promoter region by the TFSEARCH program revealed a putative CREB binding site (GTGAGCTAA) at position -113 to -105 (Fig. 1A) with a 100 point score, which is highly conserved among several mammalian species, including the mouse and rat. To evaluate the impact of this CREB motif on USP22 promoter activity, site-directed mutagenesis was introduced within the basic promoter $\mathrm{p}-210$, and the resulting plasmids were transiently transfected into the HeLa and HepG2 cells. The p-210/ CREBmut construct showed an $\sim 45 \%$ decrease in luciferase activity in both the HeLa and HepG2 cells compared to the wild-type p-210 (Fig. 1B), suggesting that the CREB site is critical for constitutive USP22 promoter activity in the cells tested.

CREB interacts with the USP22 promoter. To determine whether CREB directly binds to the USP22 promoter in vivo, chromatin immunoprecipitation (ChIP) was performed using cultured HeLa cells. Chromatin was sonicated into $400-$ 500-bp fragments and precipitated using the anti-CREB-1 antibody or rabbit IgG (negative control). The precipitated DNA was subjected to PCR using primers designed to amplify a 272-bp fragment of the USP22 promoter encompassing the CREB site. Following immunoprecipitation, the samples treated with the anti-CREB-1 antibody yielded a 272-bp PCR 
$\mathbf{A}$

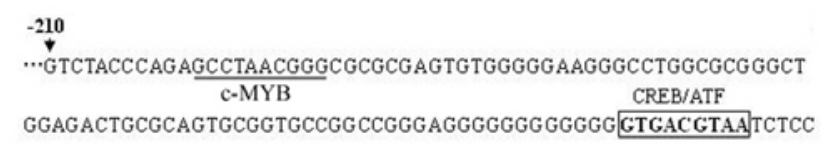

GTCCGCGGCCGCGG CGCCCCG CCCCGCGACACCCTCCG CGAACCGCGAGAGCGCAGC GCAGGCGGTCCGCAGCAGCCGCAGCTCGGGGGCGGTGCCTGCCTTGCAGCCTCCCCT CGGCGATCGCGCAGCCCCAT $\cdots$
B

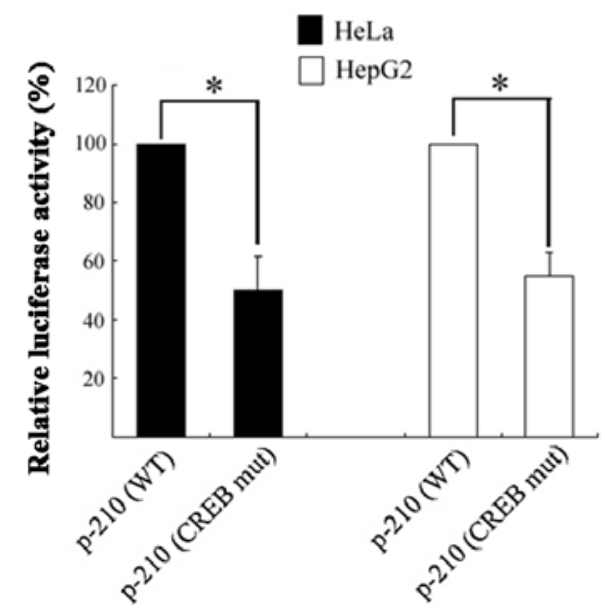

Figure 1. Effect of a CREB mutation on basal human USP22 promoter activity. (A) Sequences of the USP22 basic promoter fragment and putative transcription factor binding sites. (B) Relative luciferase expression generated by wild-ype ( -210 to +52$)$ or USP22 promoter mutants after HeLa or HepG2 cell transfection. Luciferase activities are expressed as a percentage of that noted in the wild-ype $(-210$ to +52$)$ fusion plasmid.

product, while no PCR amplification product was detected following IgG incubation (Fig. 2). These results indicate that CREB-1 constitutively occupies this region of the USP22 promoter, and thus, is likely to contribute to basal transcriptional activation.

Effect of CREB on USP22 promoter activity. To determine whether CREB is necessary for basal USP22 transcription, siRNA-mediated knockdown was employed. CREB-specific siRNA resulted in an $\sim 80 \%$ reduction in CREB protein expression relative to the control (Fig. 3A), which resulted in a significant decrease in p-210 (WT) luciferase activity, with no change in luciferase activity observed in the control group (Fig. 3B). To confirm CREB regulation of the USP22 promoter via the CREB site, HeLa cells were co-transfected with CREB siRNA and the p-210/CREBmut plasmid. This showed that siRNA-mediated knockdown had no significant impact on p-210/CREBmut promoter activity (Fig. 3C). Furthermore, the effect of CREB deletion on endogenous USP22 expression was determined by monitoring USP22 expression following CREB siRNA transfection via $\mathrm{qRT}$-PCR. The results showed that siRNA-mediated knockdown of CREB led to a significant reduction in USP22 mRNA expression levels (Fig. 3D).

Since it was now established that CREB is required for USP22 transcription, the effect of exogenous CREB on USP22

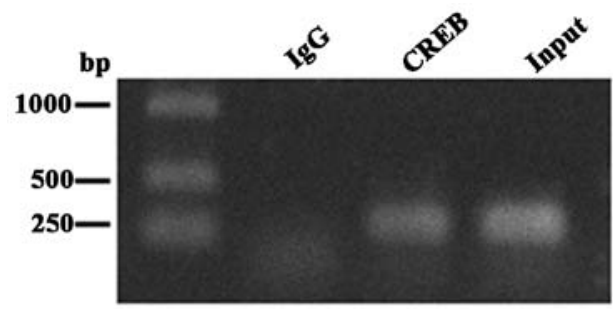

Figure 2. ChIP assays to test the binding of CREB to the USP22 promoter in vivo. Anti-CREB antibody precipitated proteins bound in vivo to the amplified sequence of the endogenous USP22 promoter, with the non-specific IgG (negative control antibody) failing to precipitate proteins. PCR products were $145 \mathrm{bp}$ and were visualized by agarose gel electrophoresis and ethidium bromide staining.

transcriptional activity was further examined through the transfection of HeLa cells with CMV-driven CREB expression plasmids pCDNA/CREB (Fig. 3E). The results showed that forced expression of exogenous CREB did not affect USP22 promoter activity (Fig. 3F). Accordingly, exogenous CREB did not promote endogenous USP22 mRNA expression (Fig. 3G). These results suggested that CREB behaves as a constitutive rather than an inducible element for USP22 transcription.

$H$-89 decreases USP22 expression via the CREB binding site. It has been reported that CREB transcriptional activity is controlled by several upstream signaling pathways. Therefore, HeLa cell USP22 promoter activity was pharmacologically targeted using $\mathrm{H}-89$, staurosporine and wortmannin to selectively inhibit PKA, PKC and PI3K, respectively. Based on published reagent concentrations (12-14), HeLa cells were treated with $30 \mathrm{nM}$ of $\mathrm{H}-89,10 \mathrm{nM}$ of staurosporine, or $10 \mathrm{nM}$ of wortmannin for 6 or $12 \mathrm{~h}$ following a 24-h transfection with $\mathrm{p}-210$. The dual luciferase assay showed that H-89, but not staurosporine or wortmannin, decreased promoter activity in a time-dependent manner (Fig. 4A), thus, indicating that the PKA signaling pathway could play a positive role in the regulation of USP22 transcription.

To determine whether H-89 suppresses endogenous USP22 expression, HeLa cells were treated with 15,30 or $60 \mathrm{nM}$ of $\mathrm{H}-89$ for $12 \mathrm{~h}$, with both USP22 mRNA and protein expression levels analyzed. As shown in Fig. 4B and C, $30 \mathrm{nM}$ of H-89 induced a very significant decrease in USP22 mRNA and protein expression in the HeLa cells. Furthermore, HeLa cells were treated with forskolin, which raises intracellular levels of cAMP. Unexpectedly, incubation with $10 \mu \mathrm{M}$ of forskolin did not increase USP22 promoter activity or endogenous mRNA expression (Fig. 4D and E). These results indicate that the PKA pathway perhaps plays a constitutive rather than an inducible role in USP22 promoter activity.

To delineate the region(s) that respond to $\mathrm{H}-89$, a series of 5' truncated promoter fragments derived from $\mathrm{p}$-210 were developed and the HeLa cells were transfected. A dual luciferase assay showed that USP22 promoter activity was serially decreased when the $5^{\prime}$ terminal of P-120 was continuously cut. Furthermore, when the $5^{\prime}$ terminal of P-120 was cut by 92 bp (P-118), a loss of $\mathrm{H}-89$ responsiveness was noted (Fig. 4F), suggesting that the $\mathrm{H}-89$ response region is located 
$\mathbf{A}$
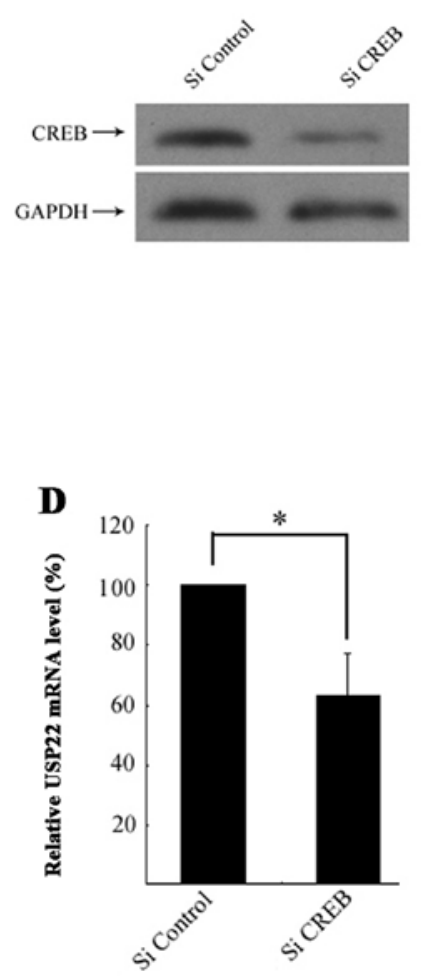

B
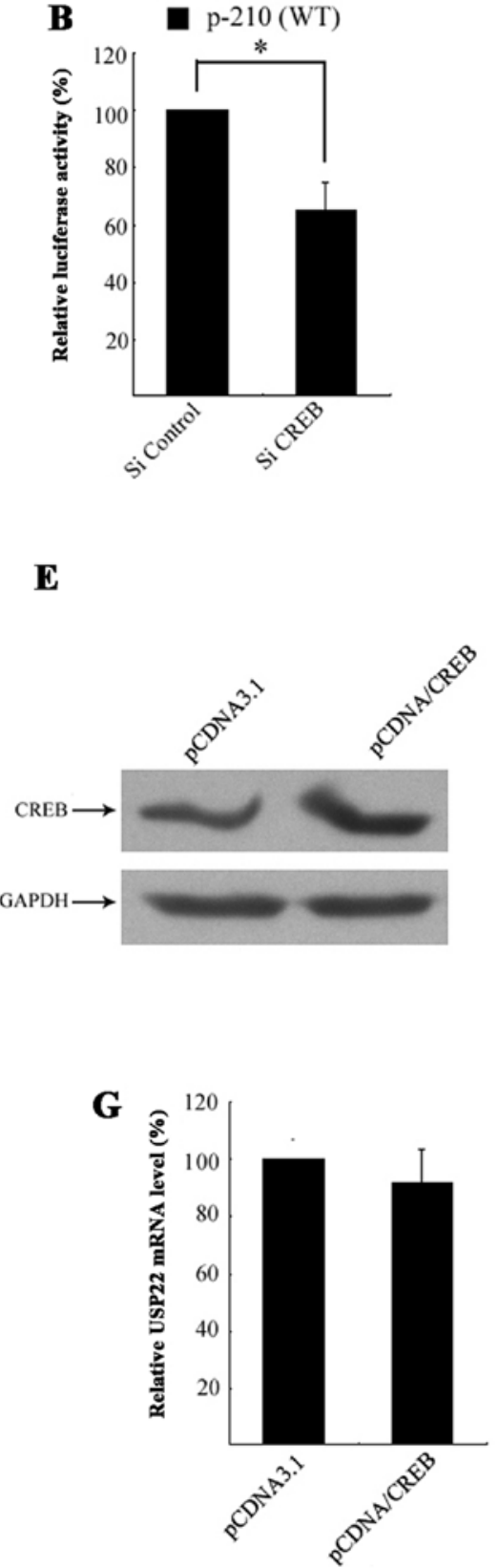

C

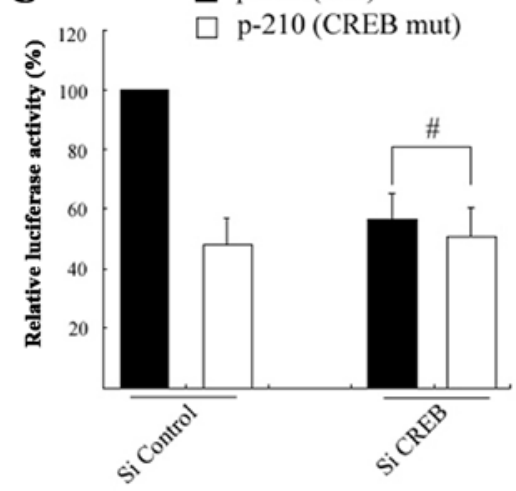

$\mathbf{F}$

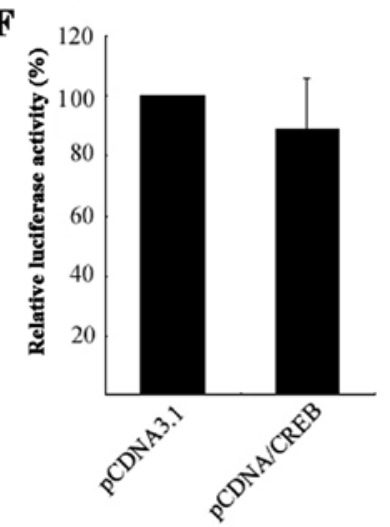

Figure 3. CREB regulates the transcription of USP22 in HeLa cells. (A) Interference efficiencies of CREB were analyzed by western blot analysis with anti-CREB and anti-GAPDH antibodies. (B) HeLa cells were transiently transfected with siRNA against CREB (CREB siRNA) and negative control siRNA. USP22 promoter activity was measured by luciferase activity $24 \mathrm{~h}$ post-transfection. Data are representative of three independent experiments $(\mathrm{P}<0.01)$. (C) HeLa cells were transiently transfected with CREB siRNA and negative control siRNA. USP22 mRNA levels were measured via qRT-PCR $24 \mathrm{~h}$ posttransfection, and the results were normalized to GAPDH. (D) HeLa cells were co-transfected with CREB siRNA and the p-210/CREBmut plasmid, and USP22 promoter activity was measured by luciferase activity $24 \mathrm{~h}$ post-transfection. (E) CREB protein were examined via western blot analysis after pCDNA/CREB was transfected into the HeLa cells. (F) HeLa cells were co-transfected with CMV-driven CREB expression plasmids (pCDNA/CREB) and USP22 promoter constructs. Luciferase activities were measured $24 \mathrm{~h}$ post-transfection. (G) HeLa cells were transiently transfected with pcDNA3.1 empty vector or pcDNA/ CREB, with USP22 mRNA quantified via qRT-PCR $24 \mathrm{~h}$ post-transfection, and the results were normalized to GAPDH.

between -129 bp and -118 bp which contains the functional CREB binding site. To determine whether $\mathrm{H}-89$ decreases USP2 2 expression via the CREB binding site, HeLa cells were transfected with wild-type p-210 or p-210/CREBmut and incubated with H-89 for $12 \mathrm{~h}$. A dual luciferase assay showed that a mutation of the CREB binding site abolished H-89 responsiveness (Fig. 4G), collectively suggesting that the PKA pathway regulates USP22 expression and its effects may be partly mediated by CREB.

\section{Discussion}

Recently, the deubiquitinating enzyme USP22 has attracted attention due to the fact that its overexpression is considered to be a hallmark of malignant cancer progression and is linked to a poor prognosis $(15,16)$. However, the mechanisms leading to USP22 transcriptional activation are still unclear. In the present study, we demonstrated that a functional CREBbinding site is required for USP22 basic promoter activity and 

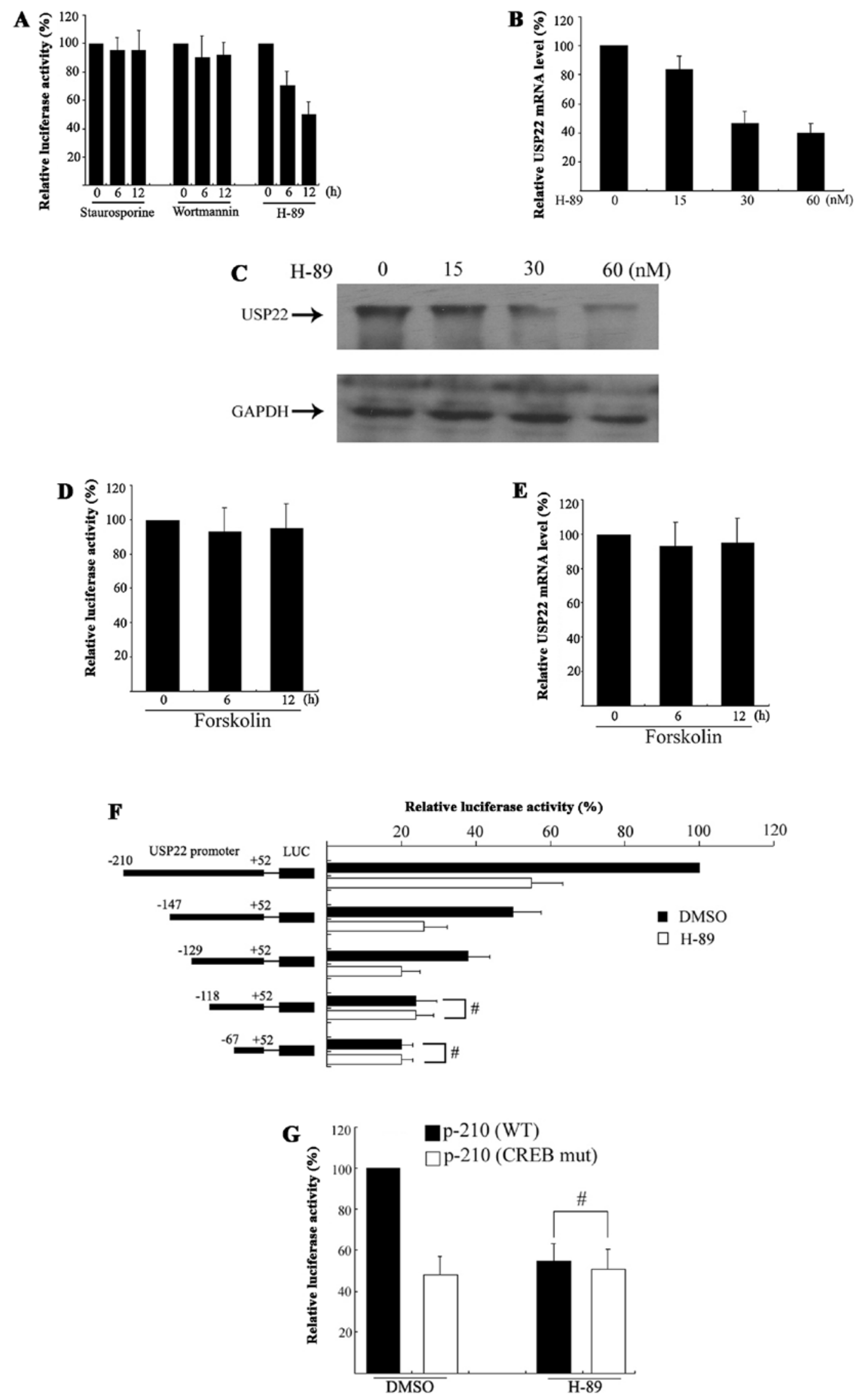

Figure 4. H-89 regulates USP22 expression in HeLa cells. (A) Luciferase assay was used to analyze the regulation of USP22 transcription in response to H-89, staurosporine and wortmannin. (B) USP22 transcriptional levels in response to H-89 were examined via qRT-PCR. Data were normalized to GAPDH and are representative of independent triplicate experiments. (C) USP22 protein expression levels in response to H-89 were examined via western blot analysis. (D) USP22 promoter activity in response to forskolin was examined via luciferase assay. (E) USP22 transcriptional levels in response to forskolin were examined via qRT-PCR. (F) 5'-deletion analysis of USP22 basic promoter activity in response to H-89 and DMSO. (G) Relative luciferase expression generated by wildtype $(-210$ to +52$)$ or the CREB binding site mutant in the presence of H-89 and DMSO. 
the CREB transcription factor binding to this site plays a positive role in constitutive transcription. Furthermore, silencing of CREB expression by siRNA, as well as PKA inhibition by H-89, decreased USP22 transcription, whereas overexpression of CREB or PKA activation by forskolin did not present enhancing effects.

Previously, we identified a 262-bp (-210 to +52$)$ region as the basic promoter for USP22 (11). Inspection of this region revealed the existence of a putative CREB consensus site consisting of the sequence GTGACGTAA at position -113 to -105. Usually, a classic CREB site consists of the palindromic sequence, TGACGTCA, and locates at 50-150 bp upstream from the start site of transcription (17). Although the putative 9-bp CREB binding site determined by TFSEARCH analysis is an atypical CREB motif, prior reports have confirmed that it is recognized and bound by CREB or other CREB/ATF members (18). In the present study, mutation of this putative CREB site resulted in a significant reduction in USP22 promoter activity, indicating that it plays a positive constitutive role in USP22 promoter activity. Therefore, delineating the transcription factor(s) binding to this site will enable a better understanding of the mechanisms responsible for USP22 expression.

In the present study, we did not characterize all the transcription factors binding to this site, but confirmed that CREB constitutively binds to this site in vitro. As a ubiquitously expressed transcription factor, CREB is involved in regulating various transcriptional genes. Many oncogenes have been found to be activated by CREB, including cyclins (19), Bcl-2 family members (20) and Egr-1 (21), with elevated CREB levels noted in several human cancers (22). Therefore, CREB is considered a key factor in mediating the malignant behavior of tumor cells. In addition to activating transcription, CREB can also mediate transcriptional repression by partnering with repressor proteins. For example, CREB acts as a negative regulator of AP- $2 \alpha$ by directly binding the AP- $2 \alpha$ promoter in melanoma cells (23). In the present study, we demonstrated that CREB plays a positive role in the regulation of USP22 transcription, with endogenous CREB knockdown resulting in a reduction in USP22 promoter activity and endogenous expression. These findings suggest that the regulation of USP22 expression by CREB may have important consequences for cancer progression.

It has been reported that CREB can stimulate both basal transcription and inducible transcription through its bipartite transcriptional activation domain (24). In this study, inhibition of CREB decreased USP22 expression, but overexpression of exogenous CREB did not enhance USP22 promoter activity, thus indicating that CREB may not mediate inducible transactivation in USP22. This may be explained by the assumption that activated CREB, rather than elevated CREB expression, plays a crucial role in USP22 transcription. Previous studies have proven that activated CREB is required for gene regulation, with this activation regulated through the phosphorylation of serine 133 by kinases such as PKA (25), PKC (26), calmodulin-dependent kinases (27) or PI3K (28). To investigate the upstream signaling pathways involved in USP22 transcriptional regulation via CREB, several kinase inhibitors were employed. The results showed that CREB functioning as a transcriptional element is controlled by PKA, due to the H-89-mediated decrease in USP22 transcription via the CREB binding site. In this study, the phosphorylation state of serine 133 following H-89 treatment was not monitored. However, previous studies have confirmed the ability of H-89 to dephosphorylate CREB in various cell types (29). Unexpectedly, USP22 promoter activity and endogenous USP22 mRNA levels were not altered in response to the PKA activator forskolin, with a similar CREB role having been previously reported. One study found that the CREB binding site in the BRCA1 proximal promoter acts as a constitutive transcriptional element (30). This constitutive transactivation role was supported by the activation or overexpression of CREB in cancers and the fact that the USP22 promoter lacks a canonical TATA box, making this type of promoter unresponsive to cAMP (31).

In summary, the present study revealed that the CREB binding site is crucial for basal USP22 promoter transcription. Furthermore, the CREB transcription factor regulates USP22 transcription by directly binding to the CREB binding site, with PKA regulation carried out through CREB. The understanding that PKA/CREB is a key regulator of human USP22 transcription may be of considerable value when selecting therapeutic targets for disease treatment.

\section{Acknowledgements}

The present study was supported by the National Nature Science Foundation of China (grant no. 31000581).

\section{References}

1. Lee HJ, Kim MS, Shin JM, Park TJ, Chung HM and Baek KH: The expression patterns of deubiquitinating enzymes, USP22 and Usp22. Gene Expr Patterns 6: 277-284, 2006.

2. Liu Y, Yang Y, Xu H and Dong X: Implication of USP22 in the regulation of BMI-1, c-Myc, p16INK4a, p14ARF, and cyclin D2 expression in primary colorectal carcinomas. Diagn Mol Pathol 19: 194-200, 2010.

3. Yang DD, Cui BB, Sun LY, et al: The co-expression of USP22 and BMI-1 may promote cancer progression and predict therapy failure in gastric carcinoma. Cell Biochem Biophys 61: 703-710, 2011.

4. Zhang Y, Yao L, Zhang X, et al: Elevated expression of USP22 in correlation with poor prognosis in patients with invasive breast cancer. J Cancer Res Clin Oncol 137: 1245-1253, 2011.

5. Zhang XY, Varthi M, Sykes SM, et al: The putative cancer stem cell marker USP22 is a subunit of the human SAGA complex required for activated transcription and cell-cycle progression. Mol Cell 29: 102-111, 2008.

6. Zhao Y, Lang G, Ito S, et al: A TFTC/STAGA module mediates histone $\mathrm{H} 2 \mathrm{~A}$ and $\mathrm{H} 2 \mathrm{~B}$ deubiquitination, coactivates nuclear receptors, and counteracts heterochromatin silencing. Mol Cell 29: 92-101, 2008.

7. Atanassov BS, Evrard YA, Multani AS, et al: Gen5 and SAGA regulate shelterin protein turnover and telomere maintenance. Mol Cell 35: 352-364, 2009.

8. Lin Z, Yang H, Kong Q, et al: USP22 antagonizes p53 transcriptional activation by deubiquitinating Sirt1 to suppress cell apoptosis and is required for mouse embryonic development. Mol Cell 46: 484-494, 2012.

9. Atanassov BS and Dent SY: USP22 regulates cell proliferation by deubiquitinating the transcriptional regulator FBP1. EMBO Rep 12: 924-930, 2011.

10. Sussman RT, Stanek TJ, Esteso P, Gearhart JD, Knudsen KE and McMahon SB: The epigenetic modifier ubiquitin-specific protease 22 (USP22) regulates embryonic stem cell differentiation via transcriptional repression of sex-determining region Y-box 2 (SOX2). J Biol Chem 288: 24234-24246, 2013.

11. Xiong J, Che X, Li X, Yu H, Gong Z and Li W: Cloning and characterization of the human USP22 gene promoter. PLoS One 7: e52716, 2012 
12. Bubis $\mathrm{M}$ and Zisapel $\mathrm{N}$ : Modulation by melatonin of protein secretion from melanoma cells: is cAMP involved? Mol Cell Endocrinol 112: 169-175, 1995.

13. Nakamura K, Bossy-Wetzel E, Burns K, et al: Changes in endoplasmic reticulum luminal environment affect cell sensitivity to apoptosis. J Cell Biol 150: 731-740, 2000.

14. Zong Y, Sun L, Liu B, et al: Resveratrol inhibits LPS-induced MAPK activation via activation of the phosphatidylinositol 3-kinase pathway in murine RAW 264.7 macrophage cells. PLoS One 7: e44107, 2012.

15. Glinsky GV: Death-from-cancer signatures and stem cell contribution to metastatic cancer. Cell Cycle 4: 1171-1175, 2005.

16. Glinsky GV: Genomic models of metastatic cancer: functional analysis of death-from-cancer signature genes reveals aneuploid anoikis-resistant, metastasis-enabling phenotype with altered cell cycle control and activated Polycomb Group (PcG) protein chromatin silencing pathway. Cell Cycle 5: 1208-1216, 2006.

17. Tinti C, Yang C, Seo H, et al: Structure/function relationship of the cAMP response element in tyrosine hydroxylase gene transcription. J Biol Chem 272: 19158-19164, 1997.

18. Euskirchen G and Snyder M: A plethora of sites. Nat Genet 36 : 325-326, 2004

19. White PC, Shore AM, Clement M, et al: Regulation of cyclin D2 and the cyclin D2 promoter by protein kinase A and CREB in lymphocytes. Oncogene 25: 2170-2180, 2006.

20. Wilson BE, Mochon E and Boxer LM: Induction of bcl-2 expression by phosphorylated CREB proteins during B-cell activation and rescue from apoptosis. Mol Cell Biol 16: 5546$5556,1996$.

21. Mayer SI, Willars GB, Nishida E and Thiel G: Elk-1, CREB, and MKP-1 regulate Egr-1 expression in gonadotropin-releasing hormone stimulated gonadotrophs. J Cell Biochem 105: 1267-1278, 2008.

22. Sakamoto KM and Frank DA: CREB in the pathophysiology of cancer: implications for targeting transcription factors for cancer therapy. Clin Cancer Res 15: 2583-2587, 2009.
23. Melnikova VO, Dobroff AS, Zigler M, et al: CREB inhibits AP-2alpha expression to regulate the malignant phenotype of melanoma. PLoS One 5: e12452, 2010.

24. Quinn PG: Distinct activation domains within cAMP response element-binding protein (CREB) mediate basal and cAMP-stimulated transcription. J Biol Chem 268: 1699917009,1993

25. Rosenberg D, Groussin L, Jullian E, Perlemoine K, Bertagna X and Bertherat J: Role of the PKA-regulated transcription factor CREB in development and tumorigenesis of endocrine tissues. Ann NY Acad Sci 968: 65-74, 2002.

26. Guo $\mathrm{Y}$ and Feng P: OX2R activation induces PKC-mediated ERK and CREB phosphorylation. Exp Cell Res 318: 2004-2013, 2012.

27. Takeda H, Kitaoka Y, Hayashi Y, et al: Calcium/calmodulindependent protein kinase II regulates the phosphorylation of CREB in NMDA-induced retinal neurotoxicity. Brain Res 1184: 306-315, 2007

28. Gibellini D, Bassini A, Pierpaoli S, et al: Extracellular HIV-1 Tat protein induces the rapid Ser133 phosphorylation and activation of CREB transcription factor in both Jurkat lymphoblastoid T cells and primary peripheral blood mononuclear cells. J Immunol 160: 3891-3898, 1998.

29. Delghandi MP, Johannessen M and Moens U: The cAMP signalling pathway activates CREB through PKA, p38 and MSK1 in NIH 3T3 cells. Cell Signal 17: 1343-1351, 2005.

30. Atlas E, Stramwasser M and Mueller CR: A CREB site in the BRCA1 proximal promoter acts as a constitutive transcriptional element. Oncogene 20: 7110-7114, 2001

31. Conkright MD, Guzman E, Flechner L, Su AI, Hogenesch JB and Montminy M: Genome-wide analysis of CREB target genes reveals a core promoter requirement for cAMP responsiveness. Mol Cell 11: 1101-1108, 2003. 